\title{
Enseñando la matemática desde la electrónica. Experiencia didáctica en la cátedra Análisis de señales y sistemas
}

\author{
Lucas Maggiolini, Ernesto Klimovsky \& José Quaglia \\ Universidad Tecnológica Nacional, Facultad Regional Paraná, Paraná, Argentina.ing.maggiolini@gmail.com,erklimo@gmail.com, \\ josequaglia1993@gmail.com
}

\begin{abstract}
Resumen-- Con el fin de aumentar la motivación de los estudiantes y reducir los índices de deserción, realizamos una experiencia didáctica que consistió en implementar trabajos prácticos de hardware (electrónica) dentro de la cátedra de Análisis de Señales y Sistemas (anteriormente Análisis Matemático III). Dichos trabajos consistieron en la aplicación directa de diversos temas matemáticos en circuitos electrónicos. Trabajamos con la Serie de Fourier, el álgebra de bloques (sistemas) y el análisis de filtros por Transformada de Laplace, en todos los casos solicitando a los estudiantes que implementen circuitos y los analicen matemáticamente.

Los resultados obtenidos apuntan a una mejora en los niveles de motivación de los estudiantes, lo que resulta en una mejora notable en los índices de deserción.
\end{abstract}

Palabras clave-- Enseñanza matemática; señales y sistemas; experiencia didáctica.

Recibido: 6 de abril de 2017. Revisado: 14 de mayo de 2017. Aceptado: 30 de mayo de 2017.

\section{Teaching mathematics from electronics. Didactic experience in System and signal analysis class}

\begin{abstract}
In order to increase students motivation and reduce dropout rates, we carried out a didactic experience that consisted in implementing practical (electronic) hardware work within the Systems and Signal Analysis class (formerly Mathematical Analysis III). These works consisted in the direct application of diverse mathematical subjects in electronic circuits. We work with the Fourier Series, block algebra (systems) and filter analysis by Laplace Transform, in all cases requiring students to implement circuits and analyze them mathematically.

The result obtained point to a greater motivation levels on the part of the students, which results in a notable improvement in the dropout rates
\end{abstract}

Keywords-- Mathematics education; systems and signals; learning experience

\section{Breve marco teórico}

Las diferentes carreras de ingeniería se caracterizan por una fuerte formación matemática, que se destaca principalmente en los primeros años del plan de estudios. Esto permite que los estudiantes posean las herramientas que permiten analizar y comprender las diferentes áreas de la especialidad. Esta característica es conocida por todos los estudiantes pero, pese a ello, es frecuente que soliciten a los docentes contenidos más orientados a la especialidad aún en cátedras específicas de matemática. Esta situación se aprecia fundamentalmente en el ciclo de formación básica donde el plan de estudios incluye pocos contenidos de la especialidad. Esto provoca en los estudiantes escasa motivación hacia el estudio de las ciencias básicas (matemática, física y química). Se presenta entonces una situación en la cual la motivación resulta del tipo extrínseca [1]. Dicho en otras palabras, los estudiantes no están motivados más allá de de la acreditación de una cátedra.

Chevallard [2] critica el paradigma de enseñanza que el denomina paradigma de la visita a obras, en el cual los estudiantes visitan y admiran obras matemáticas sin llegar a conocer sus razones de ser. Según el autor un proceso de enseñanza que posea sus fundamentos en dicho paradigma, formará en los estudiantes la creencia que el conocimiento matemático es algo de lo cual se puede prescindir casi por completo. Considerando que gran cantidad de los conceptos matemáticos analizados en las diferentes cátedras del área matemática, no poseen una aplicación directa hasta los últimos años de la carrera, surge por parte de los estudiantes la típica pregunta: “¿y esto para qué sirve?”.

Una estrategia para hacer frente a tal situación es la enseñanza de la matemática mediante la resolución de problemas. Charnay [3] es defensor de tal metodología indicando que son los problemas los que han dado origen y sentido a las matemáticas, según el autor "hacer matemática es resolver problemas" [3]. En este modelo de enseñanza el problema es el recurso por excelencia en el proceso de aprendizaje. Tal vez entonces, al utilizar problemas específicos del área correspondiente de la ingeniería, se podría aumentar la motivación de los estudiantes y lograr un mejor proceso de aprendizaje de la matemática.

\section{El problema}

Considerando la perspectiva general de una matemática orientada a la ingeniería tal cual se define en el plan de estudios, en la cátedra siempre se plantean problemas reales y aplicados a la ingeniería electrónica, como ser resolución de circuitos o cálculos de filtros. Pese a ello siempre hemos tenido la problemática de encontrarnos con estudiantes poco motivados. Aquí debemos considerar que en $3^{\circ}$ año del plan de estudios de Ingeniería en Electrónica, los estudiantes aún no han tenido 
ninguna cátedra específica de electrónica o en la cual implementen trabajos de laboratorio.

Esta falta de motivación provoca una falta de seguimiento en los contenidos de la cátedra y, remarcando que existe una fuerte interrelación de conceptos, ocurre una degradación de la matrícula durante el transcurso del año.

\subsection{Nuestra propuesta}

Con el fin de aumentar la motivación de los estudiantes y mejorar el proceso de aprendizaje implementamos diversos trabajos prácticos de hardware. Los mismos consisten en el armado de circuitos electrónicos y su análisis matemático. El procedimiento es el siguiente:

1) Solicitamos a los estudiantes que conformen grupos de trabajo (igualmente deben realizar otras actividades de la cátedra en grupo).

2) Les indicamos el circuito a implementar, qué deben analizar del mismo y proponemos algunas preguntas que permitan relacionar los aspectos electrónicos con los matemáticos.

3) Por último deben realizar una defensa oral del trabajo con el circuito funcionando. En esta instancia siempre intentamos generar debate sobre los conceptos matemáticos y su relación con la electrónica.

Debemos destacar que ya hemos realizado una experiencia similar para mejorar el seguimiento de los contenidos de la cátedra [4]. En esta nueva experiencia buscamos lograr una motivación intrínseca, con el fin que el seguimiento de los contenidos sea iniciativa de los estudiantes y no resultado de una exigencia de la cátedra.

\subsection{Trabajos implementados}

Hemos implementado tres trabajos de hardware, cada uno correspondiente a una unidad temática. El primero se desarrolla en conjunto con los conceptos de un sistema Lineal e Invariante en el Tiempo (LTI) y los conceptos de transformación de la variable independiente. El segundo se desarrolla en conjunto con la Serie y Transformada de Fourier y el análisis en frecuencia. El último se desarrolla junto con los conceptos de Laplace y filtrado.

\subsubsection{Primer trabajo de hardware}

En el primer trabajo proponemos a los estudiantes el armado de un circuito electrónico con diversos bloques que permiten analizar los conceptos que definen a un sistema LTI, el álgebra de bloques y las transformaciones de la variable independiente. El primer bloque consiste en un oscilador y un divisor de frecuencias. El oscilador genera una señal cuadrada y el divisor permite obtener 3 señales de frecuencias múltiplo de la frecuencia del oscilador. A la salida del divisor se conecta un filtro Pasa Bajos (PB) con el fin de eliminar la componente de corriente continua. Esta señal se aplica a un integrador que convierte su forma de cuadrada a triangular. El último bloque posee una llave selectora para seleccionar la señal, cuadrada o triangular, a la cual le suma un nivel definido de tensión continua y posteriormente es atenuada o amplificada.

En la Fig. 1 se aprecia un diagrama de bloques del circuito propuesto.

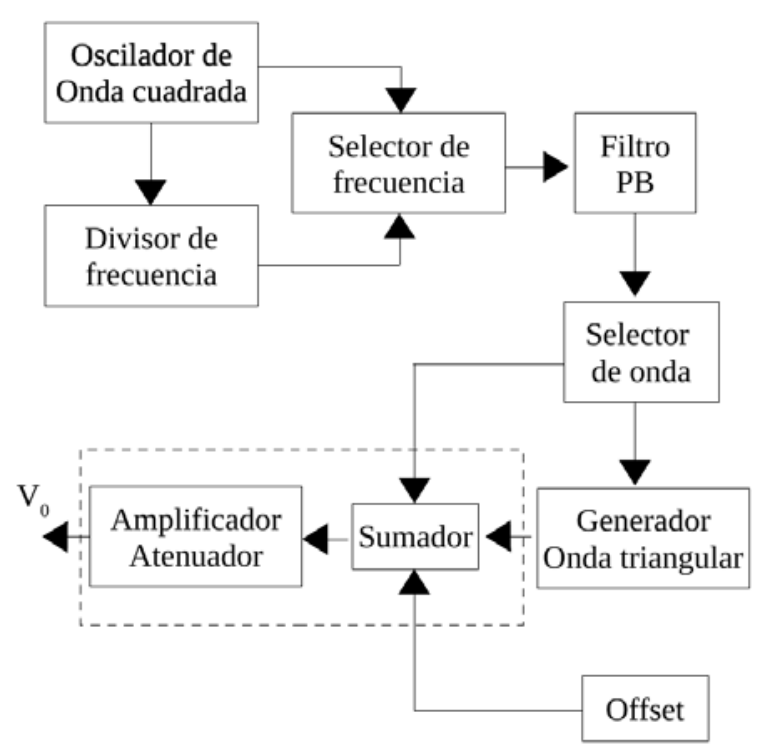

Figura 1. Diagrama de bloques del primer trabajo.

Fuente: Los autores.

La interpretación matemática y electrónica de cada bloque puede apreciarse en la Tabla 1.

Tabla 1

Interpretación Primer Trabajo

\begin{tabular}{|c|c|c|c|c|}
\hline \multicolumn{2}{|c|}{ Bloque } & \multicolumn{2}{|l|}{ Interpretación Matemática } & $\begin{array}{c}\text { Interpretación } \\
\text { Electrónica }\end{array}$ \\
\hline $\begin{array}{l}\text { Oscilador } \\
\text { Cuadrada }\end{array}$ & Onda & $x(t)=\left\{\begin{array}{c}a, 0<t<T_{0} / 2 \\
0, T_{0} / 2<t<T_{0} \\
x(t)=x\left(t+T_{0}\right)\end{array}\right\}$ & (1) & $\begin{array}{l}\text { Oscilador Astable } \\
\text { Período = T } \\
\text { Ciclo trabajo 50\% }\end{array}$ \\
\hline Divisor Frec & lencia & $y(t)=x(t / 2)$ & $(2)$ & $\begin{array}{l}\text { FlipFlop divisor } \\
\text { de frecuencia } \\
(\mathrm{J}=\mathrm{K}=1)\end{array}$ \\
\hline Filtro DC & & $H(s)=\frac{j w}{R C j w+1}$ & (3) & $\begin{array}{l}\text { Filtro RC primer } \\
\text { orden } \\
\text { Frecuencia corte } \\
1 /(\mathrm{RC})\end{array}$ \\
\hline $\begin{array}{l}\text { Generador } \\
\text { Triangular }\end{array}$ & Onda & $v_{0}(t)=\frac{-1}{R C} \int_{0}^{t} v_{i}(\tau) d \tau$ & (4) & $\begin{array}{l}\text { Amplificador } \\
\text { operacional en } \\
\text { modo integración }\end{array}$ \\
\hline Offset & & $x(t)=c$ & (5) & $\begin{array}{l}\text { Divisor resistivo } \\
\text { Tensión = C }\end{array}$ \\
\hline Sumador & & $y(t)=-x_{1-2}(t)+c$ & (6) & $\begin{array}{l}\text { Amplificador } \\
\text { operacional } \\
\text { inversor en modo } \\
\text { sumador }\end{array}$ \\
\hline $\begin{array}{l}\text { Amplificado } \\
\text { atenuador }\end{array}$ & & $y(t)=k x(t)$ & (7) & $\begin{array}{l}\text { Ajuste del valor de } \\
\text { la resistencia de } \\
\text { realimentación del } \\
\text { operacional }\end{array}$ \\
\hline
\end{tabular}

Fuente: Los autores.

Los conceptos analizados en este trabajo son:

1) Linealidad e invarianza

2) Transformaciones de la variable independiente (escalamiento) 
3) Análisis de sistemas por bloques

4) Específicamente los conceptos correspondientes a la varianza temporal se analizan en el divisor de frecuencia y los conceptos correspondientes a la linealidad se analizan en el sumador. Este trabajo está orientado a lograr en los estudiantes la apreciación de las características de un sistema LTI y cómo las mismas se manifiestan en un circuito electrónico.

En relación a la interpretación matemática, la misma se realiza en el dominio temporal o frecuencial, según facilite su análisis. El bloque correspondiente al filtro solo se menciona a los estudiantes ya que al momento de realizar este trabajo aún no se han enseñado los conceptos del análisis en frecuencia.

\subsubsection{Segundo trabajo de hardware}

En el segundo trabajo práctico presentamos a los estudiantes una señal periódica cuadrada de la cual deben calcular el desarrollo en Serie de Fourier e implementar electrónicamente las primeras tres armónicas. Posteriormente mediante un sumador realizan la suma se dichas armónicas y pueden observar la similitud de la señal obtenida con la señal propuesta.

En la Fig. 2 se aprecia la señal sobre la cual deben realizar el análisis.

El circuito posee 3 etapas principales. La primera de ellas está conformada por dos bloques, el primero consiste en un oscilador ajustado a la frecuencia $f$ que ingresa a un circuito secuencial implementado con FlipFlops. Este corresponde al segundo bloque y cumple la función de entregar 3 señales cuyas frecuencias son múltiplo de la señal generada por el reloj. Estas frecuencias corresponden a los 3 primeros armónicos de Fourier.

En la Fig. 3 se aprecia la primera etapa del segundo trabajo.

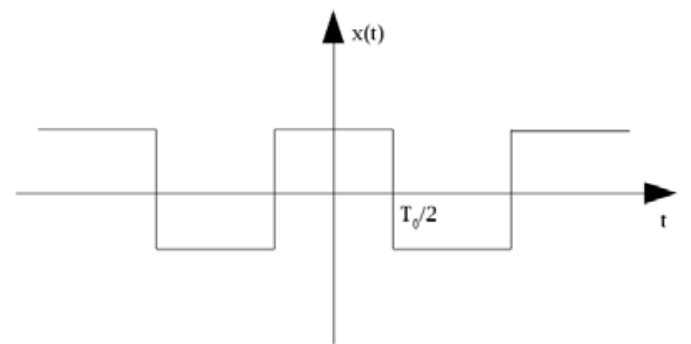

Figura 2. Señal a analizar en el segundo trabajo.

Fuente: Los autores.

Figura 3. Primera etapa del segundo trabajo.

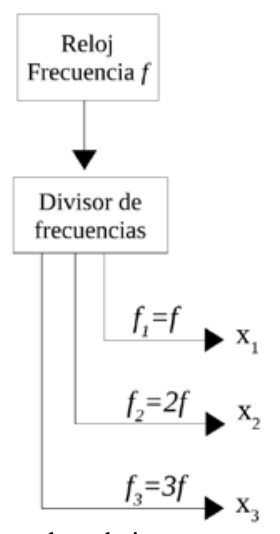

Fuente: Los autores.
La segunda etapa del circuito propuesto se repite a la salida de cada una de las 3 señales que genera el circuito secuencial. La señal de entrada es aplicada a un filtro de baja frecuencia para eliminar la componente de corriente continua, luego un filtro Paso Bajo para suavizar la señal y por último un adaptador de impedancias que permita ingresar las señales al próximo bloque.

La misma se aprecia en la Fig. 4.

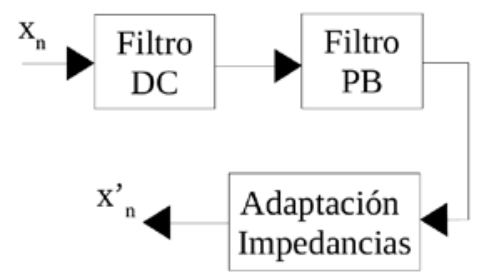

Figura 4. Segunda etapa del segundo trabajo.

Fuente: Los autores.

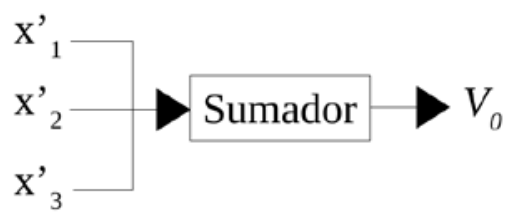

Figura 5. Tercera etapa del segundo trabajo. Fuente: Los autores.

Tabla 2

Interpretación Segundo Trabajo

\begin{tabular}{|c|c|c|c|}
\hline Bloque & Interpretación Matemática & & $\begin{array}{l}\text { Interpretación } \\
\text { Electrónica }\end{array}$ \\
\hline $\begin{array}{l}\text { Oscilador } \\
\text { Onda } \\
\text { Cuadrada }\end{array}$ & $x(t)=\left\{\begin{array}{c}a, 0<t<T_{0} / 2 \\
0, T_{0} / 2<t<T_{0} \\
x(t)=x\left(t+T_{0}\right)\end{array}\right\}$ & (8) & $\begin{array}{l}\text { Oscilador Astable } \\
\text { Período = T } \\
\text { Ciclo trabajo 50\% }\end{array}$ \\
\hline $\begin{array}{l}\text { Divisor de } \\
\text { frecuencias }\end{array}$ & $\begin{array}{c}x_{1}(t)=x(t) \\
x_{2}(t)=x(2 t) \\
x_{3}(t)=x(3 t)\end{array}$ & (9) & $\begin{array}{l}\text { Circuito } \\
\text { secuencial con } \\
\text { FliopFlop }\end{array}$ \\
\hline Filtro DC & $H(s)=\frac{1}{R C j w+1}$ & (10) & $\begin{array}{l}\text { Filtro RC primer } \\
\text { orden } \\
\text { Frecuencia corte } \\
1 /(\mathrm{RC})\end{array}$ \\
\hline Filtro PB & $H(s)=\frac{1}{(R C j w+1)^{3}}$ & (11) & $\begin{array}{l}\begin{array}{l}\text { Filtros RC en } \\
\text { cascada }\end{array}\end{array}$ \\
\hline $\begin{array}{l}\text { Adaptación } \\
\text { de } \\
\text { Impedancias }\end{array}$ & $x_{n}^{\prime}(t)=x_{n}(t)$ & (12) & $\begin{array}{l}\text { Operacional en } \\
\text { modo seguidor }\end{array}$ \\
\hline Sumador & $\begin{array}{l}V o(t)=-\left[x_{1}^{\prime}(t)+x_{2}^{\prime}(t)+\right. \\
\left.+x_{3}^{\prime}(t)\right]\end{array}$ & (13) & $\begin{array}{l}\text { Operacional } \\
\text { inversor en modo } \\
\text { sumador }\end{array}$ \\
\hline
\end{tabular}

Fuente: Los autores. 
La última etapa del circuito simplemente suma las 3 señales permitiendo obtener la señal de salida. La misma se aprecia en la Fig. 5.

Los conceptos que se analizan en este trabajo son:

Linealidad e Invarianza temporal

1) Serie de Fourier

Específicamente los conceptos relacionados a la linealidad se analizan en el bloque sumador y los conceptos relacionados a la varianza se analizan en el divisor de frecuencias. El eje principal de este trabajo se orienta al análisis de la Serie de Fourier. Aquí los estudiantes pueden apreciar cómo al sumar electrónicamente los diferentes armónicos de la serie, pueden obtener una señal considerablemente similar a la señal origen.

La interpretación matemática y electrónica de cada etapa se aprecia en la Tabla 2.

Al igual que el primer trabajo la interpretación matemática se realiza en el dominio temporal o frecuencial según se facilite su interpretación.

\subsubsection{Tercer trabajo de hardware}

En el último trabajo indicamos a los estudiantes que implementen dos filtros, uno Pasa Bajos y el otro Pasa Altos. A cada uno de ellos se aplica una señal de entrada, para luego variar su frecuencia y apreciar que ocurre a la salida. Por último deben conectar en cascada ambos filtros con el fin de lograr un filtro Pasa Banda.
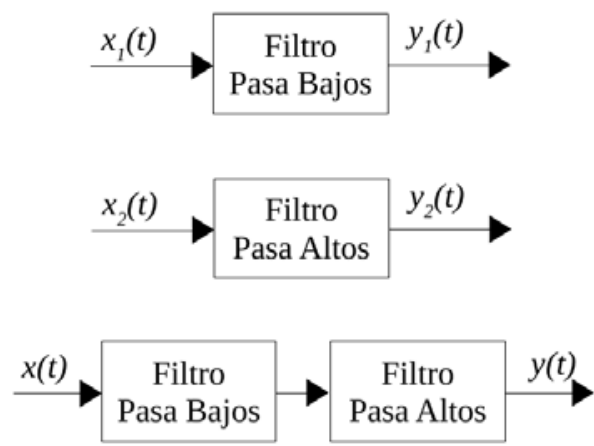

Figura 6. Diagrama en bloques del tercer trabajo. Fuente: Los autores.

Tabla 3.

Interpretación Tercer Trabajo

\begin{tabular}{|c|c|c|c|c|}
\hline \multicolumn{2}{|c|}{ Bloque } & \multicolumn{2}{|l|}{ Interpretación Matemática } & $\begin{array}{l}\text { Interpretación } \\
\text { Electrónica }\end{array}$ \\
\hline $\begin{array}{l}\text { Filtro } \\
\text { Bajos }\end{array}$ & Pasa & $H(s)=\frac{1}{R C s+1}$ & (10) & $\begin{array}{l}\text { Filtro RC primer } \\
\text { orden } \\
\text { Frecuencia } \\
1 /(\mathrm{RC})\end{array}$ \\
\hline $\begin{array}{l}\text { Filtro } \\
\text { Altos }\end{array}$ & Pasa & $H(s)=\frac{s}{\left(R^{\prime} C^{\prime} s+1\right)}$ & (11) & $\begin{array}{l}\text { Filtro RC primer } \\
\text { orden } \\
\text { Frecuencia } \\
1 /\left(\mathrm{R}^{\prime} \mathrm{C}^{\prime}\right)\end{array}$ \\
\hline
\end{tabular}

Filtro Pasa $H(s)=\frac{s}{(R C s+1)\left(R^{\prime} C^{\prime} s+1\right)} \quad$ (13) Filtros en cascada
Banda

Fuente: Los autores.
El diagrama de bloques se aprecia en la Fig. 6.

Cada uno de estos bloques deben implementarlos con filtros RC de primer orden. La interpretación matemática y electrónica de cada bloque se aprecia en la Tabla 3.

En este trabajo se analizan los conceptos de la Transformada de Laplace y su aplicación al filtrado, analizando los tres filtros básicos.

Se aprecia que a diferencia del segundo trabajo el análisis en frecuencia se realiza en el dominio de Laplace utilizando como variable $s$.

\subsection{Dificultades encontradas}

Nos encontramos con dos dificultades a superar durante la implementación de los trabajos:

1) El debate que se generó en cada grupo al momento de la entrega y defensa del trabajo, demandó más tiempo del planificado. Debido a que creemos que este debate e interpretación electrónica de la matemática es el principal aporte de los trabajos, debimos reorganizar la entrega de algunos grupos y coordinar horarios adicionales. Debemos aquí remarcar que la carga horaria de $3^{\circ}$ año de Ingeniería en Electrónica es elevada por lo cual esta coordinación de horarios resultó compleja.

2) Los estudiantes aún no poseen experiencia en la defensa oral de trabajos, por lo cual debimos orientarlos y guiarlos sobre cómo exponer los mismos, sin dejar de lado el debate sobre los conceptos clave de cada tema.

\section{Resultados}

La investigación se realizó en los estudiantes inscriptos de la cátedra Análisis de Señales y Sistemas durante los ciclos lectivos 2013, 2014, 2015 y 2016, en la Universidad Tecnológica Nacional, Facultad Regional Paraná (Argentina).

Analizamos los índices de deserción a través de la asistencia a cada parcial (tres en total) durante los 4 años mencionados. 20132014 corresponden a la metodología tradicional de enseñanza y 2015-2016 a la implementación de los trabajos de hardware.

El primer parcial se desarrolla durante el mes de mayo, el segundo parcial se desarrolla luego del receso de julio y el último en el mes de noviembre.

En la Fig. 7 se aprecia la evolución de la asistencia a la cada parcial.

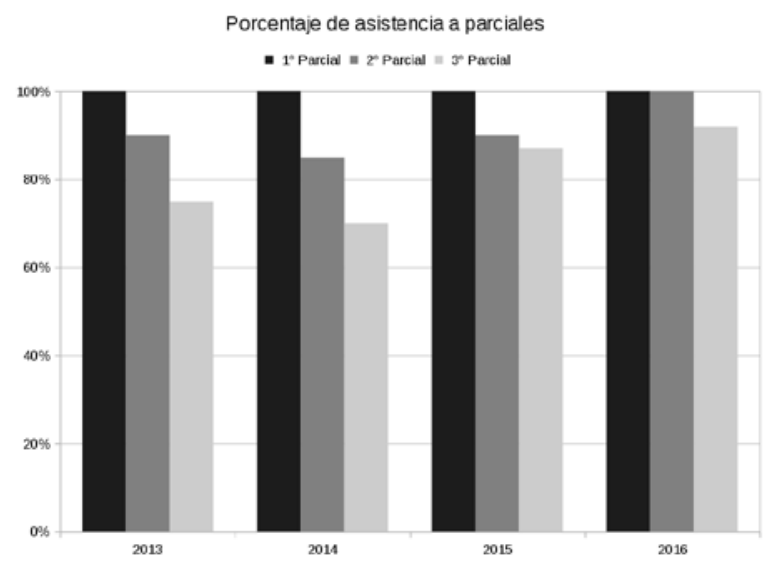

Figura 7. Índices de asistencia a parciales. Fuente: Los autores. 
Adicionalmente la cátedra realiza encuestas anuales a los estudiantes donde, entre otras cuestiones, les consulta por la implementación de los trabajos de hardware. Aproximadamente un $90 \%$ de los estudiantes reconoció como un aporte positivo la implementación de los trabajos de hardware, remarcando el aspecto motivacional al poder interactuar con componentes electrónicos y, el aspecto conceptual, al facilitar la comprensión de los contenidos más importantes de la cátedra.

\section{Discusión}

En esta experiencia abordamos la problemática relacionada a la motivación de los estudiantes frente a la enseñanza de la matemática. A tal fin implementamos en la cátedra trabajos prácticos de hardware, que consistían específicamente en el armado de circuitos electrónicos.

Se han realizado diversas experiencias que implementan la enseñanza mediante la resolución de problemas [5-6], que utilizan la modelación [7] o trabajan con prototipos y bloques [8]. En todos los casos se ha orientado la investigación hacia una mejora del proceso de enseñanza, obteniendo siempre mejoras en el aprendizaje. Solo una de las experiencias [7] destaca una mejora en la motivación de los estudiantes al permitir que ellos aprecien cuál es el fin o utilidad de los conceptos matemáticos dentro de su área de estudio.

Nuestra experiencia estuvo orientada desde un comienzo a lograr un aumento en la motivación de los estudiantes, logrando reducir considerablemente los índices de deserción como se aprecia claramente en los dos años en los cuales se implementó la metodología. La experiencia anterior realizada [4] logró aumentar el seguimiento de los contenidos de la cátedra, pero dicho logro se atribuye a una exigencia adicional de la misma, en este caso logramos aumentar el interés de estudiantes consiguiendo un cambio de motivación extrínseca a intrínseca.

\section{Conclusión}

Logramos destacar, en la experiencia realizada, que la implementación de trabajos de hardware provocó una mejora en la motivación de los estudiantes. Debimos considerar la complejidad de los trabajos desde una perspectiva electrónica, el tiempo requerido para su desarrollo y defensa, y su correlación de contenidos con la cátedra. Pese a ello los resultados justificaron ampliamente el trabajo realizado.

Considerando otras experiencias [5-8], nos encontramos actualmente planificando una implementación más profunda de los trabajos dentro de la planificación de cátedra, con el fin de lograr una mejora en la motivación pero al mismo tiempo, utilizarlos como punto de partida del proceso de enseñanza. El diseño de tales trabajos y su incorporación presentan un gran desafío ya que es compleja la labor de buscar una implementación electrónica (realizable al nivel de los estudiantes) que permita analizar cada tema o concepto de la cátedra.

\section{Bibliografía}

[1] Carretero, M., Constructivismo y educación, Buenos Aires, Argentina: Paidos, 2009, pp 85-90.

[2] Chevalard, Y., Enseñar matemáticas en la sociedad de mañana: Alegato a favor de un contraparadigma emergente, Journal of Research in Mathematics Education, 2(2), pp. 161-182, 2013.

[3] Charnay, R., Aprender (por medio de) la resolución de problemas, en Didáctica de Matemáticas, Buenos Aires, Argentina: Paidos, 1997, pp. 51-63.

[4] Klimovsky E., Maggiolini L. y Martin M., Experiencia didáctica en la cátedra análisis de señales y sistemas, Revista Argentina de Enseñanza de la Ingeniería, 4(9), pp. 51-57, Septiembre, 2015.

[5] Sanchez, J., Influencia en la enseñanza de la matemática basada en la resolución de problemas en el mejoramiento del rendimiento académico, Tesis, Maestría en Educación, Universidad Nacional Mayor de San Marcos, Lima, Perú, 2009.

[6] Bravo-Bohórquez A., Castañeda-Rodriguez L., Hernández-Yomayusa H. y Hernández-Hernández L., Enseñanza de las matemáticas en ingeniería: Modelación matemática y matemática contextual, Revista Educación en Ingeniería, 11(21), pp. 27-31. Marzo, 2016.

[7] Peña-Páez L. y Morales-García J., La modelación matemática como estrategia de enseñanza-aprendizaje: El caso del área bajo la curva, Revista Educación en Ingeniería, 11(21), pp. 64-71, Marzo, 2016.

[8] Lopez-Santos O., Cambiando el rol de las actividades experimentales en la enseñanza de Electrónica de potencia, Revista Educación en Ingeniería, 8(15), pp. 12-23, Junio, 2013.

L. Maggiolini, recibió el título de Ing. en Electrónica en 2009 en la Facultad Regional Paraná de la Universidad Tecnológica Nacional, Argentina. En 2012 recibió el título de Profesor de Enseñanza Técnica en la Escuela de Educación Técnica N. ${ }^{\circ} 1$ de Nogoyá. En 2014 recibió el título de Esp. en Docencia Universitaria. Actualmente es jefe de trabajos prácticos en la Universidad Tecnológica Nacional, Regional Paraná, Argentina.

ORCID: 0000-0002-0360-397X

E. Klimovsky, recibió el título de Ing. en Electrónica en 1996 en la Facultad Regional Paraná de la Universidad Tecnológica Nacional, Argentina. En 2004 recibió el título de Dr. en Tecnología Química de la F.I.Q. de la U.N.L. Actualmente es profesor asociado en la Universidad Tecnológica Nacional, Regional Paraná, Argentina. ORCID: 0000-0003-4659-8004.

J. Quaglia, es estudiante avanzado de la carrera de Ingeniería en Electrónica en la Universidad Tecnológica Nacional, Regional Paraná, Argentina. ORCID: 0000-0002-4784-4054 